\title{
Should oral anticoagulation be discontinued after 3 months in the setting of a first high- risk pulmonary embolism secondary to a major transient/reversible risk factor?
}

\author{
To the Editor:
}

Should oral anticoagulation be discontinued after 3 months in the setting of a first high-risk pulmonary embolism event secondary to a major transient/reversible risk factor?

Important recommendations for the regimen and duration of anticoagulation after pulmonary embolism (PE) are proposed in the new/revised concepts in the 2019 European Society of Cardiology (ESC)/ European Respiratory Society (ERS) guidelines for the diagnosis and management of acute pulmonary embolism. Potential indications for extended anticoagulation, after initial therapeutic anticoagulation for at least 3 months, depend on the presence of risk factors.

For patients with first pulmonary embolism (PE)/venous thromboembolism (VTE) secondary to a major transient/reversible risk factor, discontinuation of therapeutic oral anticoagulation is recommended after 3 months [1].

This is plausible as the risk of recurrence in this subgroup with a major reversible risk factor is low, as only estimated to be $1 \%$ within 1 year and $3 \%$ within 5 years after stopping therapy in VTE provoked by surgery as a major transient risk factor $[2,3]$.

However, does this recommendation remain valid for patients surviving high-risk PE who have been treated with primary reperfusion treatment, usually systemic thrombolysis, or intermediate-risk PE who developed signs of haemodynamic instability needing rescue reperfusion therapy during the first 2-3 days? The index case raising this question was a 53-year-old woman who survived out-of-hospital cardiac arrest attributed to massive pulmonary embolism in the setting of a recent major transient risk factor.

The Management Strategy and Prognosis of Pulmonary Embolism (MAPPET) registry revealed a high in-hospital mortality according to the degree of haemodynamic compromise in 1001 patients with acute $\mathrm{PE}$, being $8.1 \%$ in the presence of right ventricular dysfunction, $24.5 \%$ if cardiogenic shock and $64.8 \%$ after cardiopulmonary resuscitation. In this registry, a major transient/reversible risk factor was not uncommon, with a reported incidence of recent major operation (within 10 days) in 274 (27\%) or a recent major trauma or fracture (within 10 days) in 106 (11\%) cases [4].

Data on the impact of extended oral anticoagulant therapy after a first episode of pulmonary embolism is scarce. Only two randomised controlled trials have addressed the question of long-term anticoagulation in patients with PE [5, 6]. In the study of AGNeLL et al. [6], 31 patients (9\%) had initially received thrombolytic therapy, but further specific data on recurrence in this subgroup is absent. In the Pulmonary Embolism Thrombolysis (PEITHO) trial (1006 patients), a randomised, double-blind trial, comparing tenecteplase plus heparin with placebo plus heparin in normotensive patients with intermediate-risk pulmonary embolism, surgery or major trauma in the month before the index event was present in $6 \%$ of patients [7]. In a post hoc analysis of post-PE impairment in survivors of acute intermediate-risk PE, neither duration nor type of treatment after the initial phase was specified [8].

@ERSpublications

Oral anticoagulation should be discontinued after 3 months for a first episode of pulmonary embolism secondary to a major transient/reversible risk factor, nevertheless some caution is needed in patients presenting with high-risk pulmonary embolism http://bit.ly/2NIdmQl

Cite this article as: Slabbynck H, Clukers J, Galdermans D. Should oral anticoagulation be discontinued after 3 months in the setting of a first high-risk pulmonary embolism secondary to a major transient/ reversible risk factor? Eur Respir J 2020; 55: 1902028 [https://doi.org/10.1183/13993003.02028-2019]. 
Despite the fact that distal and proximal deep vein thrombosis (DVT) and PE are manifestations of the same thromboembolic disease, the phenotypic expression seems to be predetermined.

In a patient-level meta-analysis of more than 2500 unselected patients recruited into prospective cohort studies, the overall 5-year risk of a recurrent VTE (DVT or PE) was similar in patients initially presenting with either PE or proximal DVT (22\% versus 26.4\%), but on the other hand the 5-year risk of recurrence as PE was three-fold higher after initial PE than it was after initial proximal DVT (10.6 versus 3.6\%) [9].

There seems to be a two- to three-fold increase in case fatality rates of recurrent VTE in patients who presented with pulmonary embolism compared with patients who presented with DVT [1].

In the PADIS-PE (Prolonged Anticoagulation During 18 months versus placebo after Initial Six-month treatment for a first episode of idiopathic Pulmonary Embolism) trial, researchers assessed risk factors for recurrent venous thromboembolism after unprovoked pulmonary embolism. A pulmonary vascular obstruction index $\geqslant 40 \%$ at diagnosis (present in $40 \%$ of patients) was associated with a two-fold increased risk of recurrence [10].

As stated in the updated ESC/ERS guidelines, oral anticoagulants are highly effective in preventing recurrent VTE during treatment, but they do not eliminate the risk of subsequent recurrence after the discontinuation of treatment. The bleeding risk of these treatments should be considered to determine which patients are eligible candidates for extended or indefinite anticoagulation.

In conclusion, data on the risks and benefits of long-term anticoagulation in patients with a major transient/reversible risk factor surviving reperfusion therapy for a first episode of high-risk PE or deteriorating intermediate-risk PE are lacking. Although recurrence of PE in patients with major transient/ reversible risk factor is uncommon, the phenotype of the initial event in this population is associated with a high mortality. In this specific population there is a need for more data confirming the safety of discontinuing therapeutic oral anticoagulation after 3 months, or data supporting thorough predictive risk assessment for patients that would benefit from indefinite treatment.

Hans Slabbynck, Johan Clukers 10 and Daniella Galdermans

Dept of Respiratory Medicine, ZNA Middelheim, Antwerp, Belgium.

Correspondence: Hans Slabbynck, ZNA Middelheim, Dept of Pulmonology, Lindendreef 1, 2020 Antwerp, Belgium. E-mail: hans.slabbynck@zna.be

Received: 15 Oct 2019 | Accepted after revision: 27 Oct 2019

Authors contributions: All authors contributed equally to literature search, designing and writing of the manuscript.

Conflict of interest: None declared.

\section{References}

1 Konstantinides SV, Meyer G, Becattini C, et al. 2019 ESC Guidelines for the diagnosis and management of acute pulmonary embolism developed in collaboration with the European Respiratory Society (ERS): The Task Force for the diagnosis and management of acute pulmonary embolism of the European Society of Cardiology (ESC). Eur Respir J 2019; 54: 1901647.

2 Kearon C, Akl EA, Ornelas J, et al. Antithrombotic therapy for VTE disease: CHEST guideline and expert panel report. Chest 2016; 149: 315-352.

3 Iorio A, Kearon C, Filippucci E, et al. Risk of recurrence after a first episode of symptomatic venous thromboembolism provoked by a transient risk factor: a systematic review. Arch Intern Med 2010; 170: 1710-1716.

4 Kasper W, Konstantinides S, Geibel A, et al. Management strategies and determinates of outcome in acute major pulmonary embolism: results of a multi-center registry. J Am Coll Cardiol 1997; 30: 1165-1171.

5 Couturaud F, Sanchez O, Pernod G, et al. Six months vs extended oral anticoagulation after a first episode of pulmonary embolism: the PADIS-PE randomized clinical trial. JAMA 2015; 314: 31-40.

6 Agnelli G, Prandoni P, Becattini C, et al. Extended oral anticoagulant therapy after a first episode of pulmonary embolism. Ann Intern Med 2003; 139: 19-25.

7 Meyer G, Vicaut E, Danays T, et al. Fibrinolysis for patients with intermediate-risk pulmonary embolism. $N$ Engl J Med 2014; 370: 1402-1411.

8 Barco S, Russo M, Vicaut E, et al. Incomplete echocardiographic recovery at 6 months predicts long-term sequelae after intermediate-risk pulmonary embolism. A post-hoc analysis of the Pulmonary Embolism Thrombolysis (PEITHO) trial. Clin Res Cardiol 2019; 108: 772-778.

9 Baglin T, Douketis J, Tosetto A, et al. Does the clinical presentation and extent of venous thrombosis predict likelihood and type of recurrence? A patient level meta-analysis. J Thromb Haemost 2010; 8: 2436-2442.

10 Tromeur C, Sanchez O, Presles E, et al. Risk factors for recurrent venous thromboembolism after unprovoked pulmonary embolism: the PADIS-PE randomised trial. Eur Respir J 2018; 51: 1701202. 


\section{From the authors:}

We would like to thank H. Slabbynck and co-workers for their interest in the 2019 European Society of Cardiology/European Respiratory Society guidelines on the diagnosis and management of acute pulmonary embolism (PE), and for their interesting comment on the treatment duration of patients with a high-risk PE secondary to a major transient/reversible risk factor.

Patients with a PE not related to a major transient provoking factor have a 8 to $10 \%$ cumulative recurrence risk 1 year after the end of anticoagulant treatment. In these patients, the initial pulmonary vascular obstruction is associated with an increased risk of residual pulmonary vascular obstruction at 6 months, and patients with a residual vascular obstruction have a higher risk of recurrent PE [1,2]. On the other hand, patients with PE provoked by a major transient risk factor, such as surgery, have a much lower risk of recurrence after the end of anticoagulant treatment, and this is why guidelines recommend 3 months of anticoagulant treatment $[3,4]$. For these patients, we are not aware of any data suggesting that either the initial vascular obstruction or the severity of the initial PE may be associated with the risk of recurrence. In addition, there are no data to suggest that the severity of the initial episode may predict the severity of a possible recurrent event. Because of this, the guidelines task force agreed to recommend a short, 3-month treatment duration of anticoagulation after PE provoked by a major provoking factor, regardless of the severity of the index episode [5]. We do acknowledge that, in clinical practice, when a high-risk, life-threatening PE has been suffered, this results in persisting uncertainty and fears for the patients and their families, and that the wish of some of these patients to prolong anticoagulation needs to be taken into account in the decision. In such cases, the questionable benefits of continuing anticoagulation should be explained to the patient along with the bleeding risk of prolonging this treatment.

@ERSpublications

For patients with first pulmonary embolism secondary to a major transient/reversible risk factor, discontinuation of therapeutic oral anticoagulation is recommended after 3 months http://bit.ly/2Pp68Ti

Cite this article as: Meyer G, Konstantinides S. Should oral anticoagulation be discontinued after 3 months in the setting of a first high-risk pulmonary embolism secondary to a major transient/reversible risk factor? Eur Respir J 2020; 55: 1902323 [https://doi.org/10.1183/13993003.02323-2019].

Guy Meyer ${ }^{1}$ and Stavros Konstantinides $\circledast^{2,3}$

${ }^{1}$ Division of respiratory disease, APHP Centre; Université Paris Descartes; Université de Paris, Paris, France. ${ }^{2}$ Center for Thrombosis and Haemostasis, University Medical Centre Mainz, Mainz, Germany. ${ }^{3}$ Dept of Cardiology, Democritus University of Thrace, Alexandroupolis, Greece.

Correspondence: Guy Meyer, Service de Pneumologie, Hôpital Européen Georges Pompidou, 20 rue Leblanc, 75015 Paris, France. E-mail: guy.meyer@aphp.fr

Received: 04 Dec 2019 | Accepted: 10 Dec 2019

Conflict of interest: G. Meyer reports grants and non-financial (travel) support from Leo Pharma, BMS-Pfizer, Stago and Bayer Healthcare, outside the submitted work. S. Konstantinides reports grants and personal fees from Boehringer Ingelheim, Daiichi-Sankyo, Bayer AG, Biocompatibles Group UK andActelion, personal fees from BMS-Pfizer and MSD, outside the submitted work.

\section{References}

1 Raj L, Robin P, Le Mao R, et al. Predictors for Residual Pulmonary Vascular Obstruction after Unprovoked Pulmonary Embolism: Implications for Clinical Practice-The PADIS-PE Trial. Thromb Haemost 2019; 119: 1489-1497.

2 Tromeur C, Sanchez O, Presles E, et al. Risk factors for recurrent venous thromboembolism after unprovoked pulmonary embolism: the PADIS-PE randomised trial. Eur Respir J 2018; 51: 1701202.

3 Baglin $\mathrm{T}$, Luddington R, Brown $\mathrm{K}$, et al. Incidence of recurrent venous thromboembolism in relation to clinical and thrombophilic risk factors: prospective cohort study. Lancet 2003; 362: 523-526.

4 Kearon C, Akl EA, Comerota AJ, et al. Antithrombotic therapy for VTE disease: Antithrombotic Therapy and Prevention of Thrombosis, 9th Edn: American College of Chest Physicians Evidence-Based Clinical Practice Guidelines. Chest 2012; 141: e419S-e494S.

5 Konstantinides SV, Meyer G, Becattini C, et al. 2019 ESC Guidelines for the diagnosis and management of acute pulmonary embolism developed in collaboration with the European Respiratory Society (ERS): The Task Force for the diagnosis and management of acute pulmonary embolism of the European Society of Cardiology (ESC). Eur Respir J 2019; 54: 1901647. 